\title{
EVIDENCIAS EPIDEMIOLÓGICAS DA OCORRENCIA DE ESCABIOSE, EM HUMANOS, CAUSADA PELO SARCOPTES SCABIEI (DEGEER, 1778) VAR. CANIS (BOURGUIGNON, 1853)*
}

\author{
Maria Helena Matiko Akao Larsson**
}

RSPUB $9 / 420$

\begin{abstract}
LARSSON, M. H. M. A. Evidências epidemiológicas da ocorrência de escabiose, em humanos, causada pelo Sarcoptes scabiei (DeGeer, 1778) var. canis (Bourguignon, 1853). Rev. Saúde públ., S. Paulo, $12: 333-9,1978$.
\end{abstract}

RESUMO: A partir de 27 cães com sarna sarcóptica, envolvendo 143 pessoas expostas à infestação, observaram-se $58(40,56 \%)$ com lesões cutâneas sugestivas de escabiose. Tais lesōes mostraram-se mais incidentes nas mulheres do que nos homens e individuos de todas as faixas etárias foram acometidos, indistintamente. Foi demonstrada a presença do agente em 3 dos 12 casos humanos observados que mantiveram contato com animais escabiosos.

Unitermos: Escabiose. Cães. Sarcoptes scabiei.

\section{N T RODUCAO}

A escabiose humana de origem canina, dermatite causada pelo ácaro Sarcoptes scabiei (DeGeer, 1778) caracteriza-se por prurido intenso e lesões cutâneas, representadas por pápulas e escoriações, que apresentam localização diversa daquela da escabiose clássica. Esta dermatite, também conhecida como sarna acomete o homem e outros mamíferos, independente de climas ou regiōes.

Para cada espécie animal existe uma variedade de Sarcoptes scabiei. Nenhuma diferença morfológica entre as diferentes variedades tem sido descrita, mas as diferenças biológicas pa:e`em existir, tanto que - ácaro do equino (S. scabiei var. equi), embora capaz de parasitar o homem, não produz, usualmente, uma infestação permanente (Toomey24, 1922; Mellanby 14, 1943; Baker e Wharton ${ }^{1}$, 1952; Tannenbaum ${ }^{22}$, 1965).

Em 1673, Sauvages fez a primeira menção de que a sarna sarcóptica do cão podia ser transmitida ao homem. No decorrer do século passado numerosos relatos de escabiose humana de origem canina foram relacionados na literatura européia (Smith e Claypoole $\left.{ }^{20}, 1967\right)$.

Toomey ${ }^{24}$ (1922) e Friedmann ${ }^{11}$ (1936) realizaram trabalhos de revisão de escabiose animal no homem; porém, nos tratados de dermatologia as referências relativas ao assunto são muito limitadas (Baker e col. ${ }^{2}, 1956$; Beck $\left.{ }^{3}, 1965\right)$.

* Resumo da dissertação apresentada à Comissão de Concurso de Mestrado em Saúde Pública, no Departamento de Epi lemiologia da Faculdade de Saúde Pública da USP.

* Do Departamento de Patologia e Clinica Médicas da Faculdade de Medicina veterinária e Zootecnia da USP - Cidade Universitária - 05508 - São Paulo, SP - Brasil. 
LARSSON, M. H. M. A. Evidências epidemiológicas da ocorrência de escabiose, em humanos, causada pelo Sarcoptes scabiei (DeGeer, 1778) var. canis (Bourguignon, 1853). Rev. Saúde públ., S. Paulo, $12: 333-9,1978$.

A escabiose humana de origem animal, apesar de sua curta evolução, é motivo de grande desconforto (Tannenbaum 22,1965 ). Nos últimos anos, despertou-se o interesse sobre esta entidade, particularmente quando causada pela variedade canina (Emde ${ }^{9}$, 1961; Tannenbaum 22, 1965; Smith e Claypoole ${ }^{20}, \quad 1967$; Thomsett 23, 1968; Norins ${ }^{16}, 1969$; Elgart e Higdon 8, 1972; Richardson 19, 1972; Svartman e col. 21, 1972; Charlesworth e Johson 6, 1974).

A sarna sarcóptica no cão caracteriza-se pelo aparecimento de pequenas pápulas esbranquiçadas ou eritematosas que se localizam, inicialmente, nas regiões inguinais, axilares e bordos das orelhas. Vesículas também podem ser visualizadas no início do processo, mas logo são substituídas por escoriações e crostas. O quadro é acompanhado por intenso prurido e perda de pelos; a piodermite secundária é de observação frequente (Smith e Claypoole 20) 1967; Muller e Kirk 15, 1969; Buell 5, 1973).

A sarna sarcóptica do cão afetando o homem é caracterizada, ainda, pela dificuldacle de encontrar o agente nas descamaçĩes cutâneas.

Tendo em vista a importância da escabiose humana de origem canina tanto en Clínicas Médicas Humana e Veterinária quanto em Saúde Pública, pois se trata de uma antropozoonose, resolvemos realizar um estudo sobre casos de escabiose humana de origem animal, salientando alguns aspectos epidemiológicos.

\section{MATERIAL E METODOS}

o presente estudo foi realizado com base nos casos de escabiose canina, atendidos pela disciplina de Patologia e Clínica Médica I (Monogástricos) da Faculdade de Medicina Veterinária e Zootecnia da USP, no período de fevereiro de 1975 a janeiro de 1976.

O diagnóstico da parasitose nos animais foi feito com base nos sintomas e confirmado pelo encontro e identificação do agente em raspados de pele.
A identificação do agente foi realizada pelo exame do raspado de pele; ao material, colocado sobre lâminas, foram adicionadas 1 ou 2 gotas de hidróxido de potássio a $10 \%$ e pesquisado ao microscópio óptico com aumento de $100 \mathrm{x}$ e/ou $400 \mathrm{x}$.

Uma vez estabelecido o diagnóstico de escabiose no animal, o proprietário fornecia, além dos dados relativos à sua identificação completa, outros referentes a: classe sócioeconômica, número de pessoas que mantiveram contacto com o animal, número de pessoas com lesões cutâneas sugestivas da doença, idade e sexo das mesmas. Foram colhidas, ainda, informações relativas à evolução das lesões dermatológicas dos proprietários, após tratamento especifico preconizado por especialistas.

Após 12 meses de pesquisa, somanos 27 casos de sarna sarcóptica em cães, envolvendo um total de 58 pessoas com prováveis sintomas de escabiose. Destes 58 individuos, 12 foram submetidos ao exame de raspado de pele, sendo que em 3 deles conseguimos demonstrar a presença do parasita. Estes exames, em humanos, foram realizados sob a orientação de um profissional.

\section{RESULTADOS}

\section{Escabiose em animais}

a. Sexo:

Animais de ambos os sexos foram acotidos indiferentemente, como mostra a Tabela 1 (estatisticamente não significante ao nivel de $\alpha=0,05$ ).

\section{TA B E L A 1}

Casos de sarna sarcóptica em cães atendidos na Faculdade de Medicina Veterinária e Zootecnia da USP, segundo o sexo, no perfodo de fevereiro de 1975 a janeiro de 1976.

\begin{tabular}{lcc}
\hline Sexo & no de casos & $\%$ \\
\hline Fêmea & 14 & 51,8 \\
Macho & 13 & 48,2 \\
\hline Total & 27 & 100,0 \\
\hline
\end{tabular}


LARSSON, M. H. M. A. Evidências epidemiológicas da ocorrência de escabiose, em humanos, causada pelo Sarcoptes scabiei (DeGeer, 1778) var. canis (Bourguignon, 1853). Rev. Saúde públ. S. Paulo, 12:333-9, 1978 .

\section{b. Idade:}

A incidência foi maior em animais jovens do que em adultos, como se verifica na Tabela 2 .

\section{T A B E L A 2}

Casos de sarna sarcóptica em cães atendidos na Faculdade de Medicina Veterinária e Zootecnia da USP, segundo a idade, no período de fevereiro de 1975 a janeiro de 1976.

\begin{tabular}{rrrr}
\hline Idade em anos & no de casos & no casos/idade \\
\hline $0 \quad-\quad 1$ & 13 & 13,0 \\
$1-1-$ & 7 & 5 & 1,7 \\
5 & -10 & 1 & 1,0 \\
$10 \quad-15$ & 1 & 0,2 \\
15 & -20 & & 0,2 \\
\hline
\end{tabular}

\section{¿. Variação sazonal:}

Quanto à variação sazonal os resultados obtidos não foram conclusivos, conforme se visualiza na Fig. 1.

Lesões cutâneas, em humanos, sugestivas de escabiose canina.

a. Número de pessoas com lesões dermatológicas sugestivas de escabiose:

De um total de 143 pessoas que mantiveram contacto com animais doentes, 58 $(40,56 \%)$ apresentaram lesões de pele semelhantes às da escabiose.

b. Sexo:

Os individuos do sexo feminino foram afetados com maior frequiência que os do

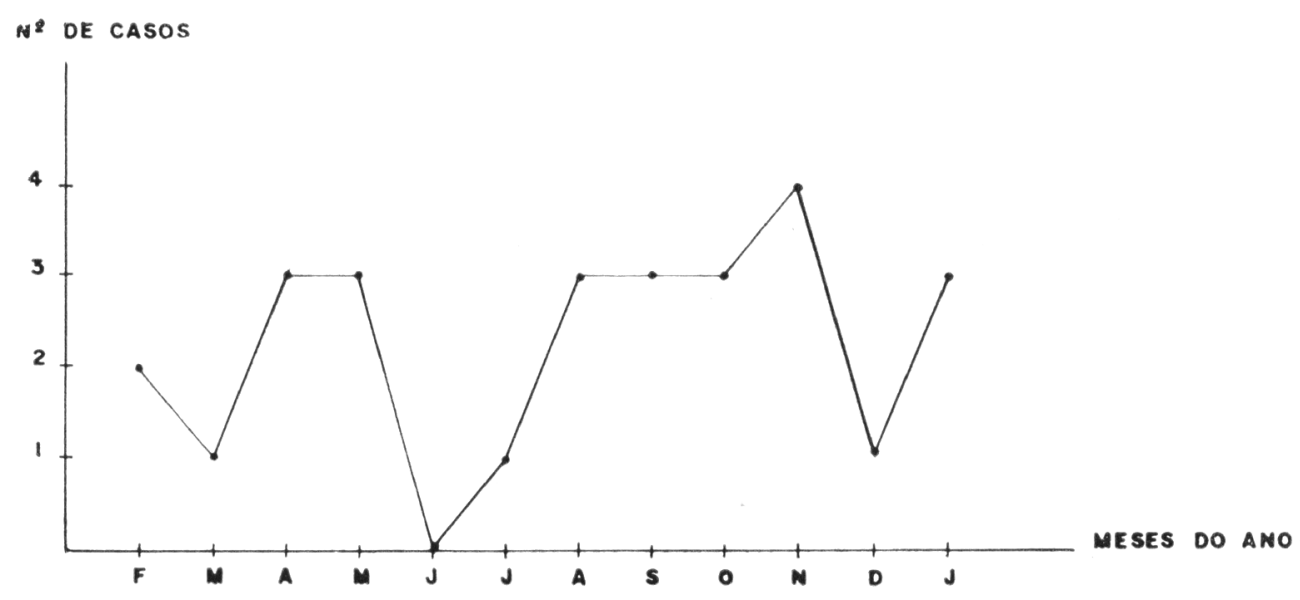

Fig. 1 - Casos de sarna sarcóptica em căes atendidos na Faculdade de Medicina Veterinária e Zootecnica da USP, no periodo de fevereiro de 1975 a janeiro de 1976

sexo masculino, como se nota na Tabela 3 (estatisticamente significante ao nível de $\alpha=0,05)$

\section{c. Idade:}

A suscetibilidade foi observada em todas as faixas etárias, como mostra a Tabela 4.
T A B E L A 3

Casos em humanos com lesões sugestivas de escabiose de origem canina, segundo sexo. São Paulo, fevereiro de 1975 a janeiro de 1976.

\begin{tabular}{lcc}
\hline Sexo & no de casos & $\%$ \\
\hline Feminino & 37 & 63,8 \\
Masculino & 21 & 36,2 \\
\hline Total & 58 & 100,0 \\
\hline
\end{tabular}


LARSSON, M. H. M. A. Evidéncias epidemiológicas da ocorrência de escabiose, em humanos, causada pelo Sarcoptes scabiei (DeGeer, 1778) var. canis (Bourguignon, 1853). Rev. Saúde públ., S. Paulo, $12: 333-9$, 1978.

\section{TA B ELA 4}

Casos em humanos com lesões sugestivas de escabiose de origem canina, segundo i zade. São Paulo, fevereiro de 1975 a janeiro de 1976.

\begin{tabular}{ccc}
\hline Idade em anos & ni de casos & n' casos/ida Le \\
\hline $0-1$ & 1 & 1,00 \\
$1-5$ & 3 & 0,75 \\
$5-10$ & 7 & 1,40 \\
$10-15$ & 9 & 1,80 \\
$15-20$ & 4 & 0,80 \\
$20-25$ & 8 & 1,60 \\
$25-30$ & 3 & 0,60 \\
$30-35$ & 4 & 0,80 \\
$35-40$ & 5 & 1,00 \\
$40-45$ & 8 & 1,60 \\
$45-50$ & 4 & 0,80 \\
$50-55$ & -6 & - \\
$55-60$ & 2 & 0,40 \\
\hline
\end{tabular}

\section{d. Incidência anual:}

Houve incidência flutuante durante os meses do ano, ora com freqüências elevadas, ora com freqüência nula, como mostra a Fig. 2.

\section{e. Classe sócio-econômica:}

O Serviço de Triagem do Ambulatório da Faculdade de Medicina Veterinária e Zootecnia da USP adota uma classificação sócio-econômica dos proprietários dos animais atendidos, que se baseia em dados como: renda familiar, profissão, número de pessoas da familia que trabalham, propriedade de imóvel e/ou veículo automotor. Em ordem decrescente a classificação é a seguinte: $\mathrm{A}, \mathrm{B}, \mathrm{C}$ e $\mathrm{D}$.

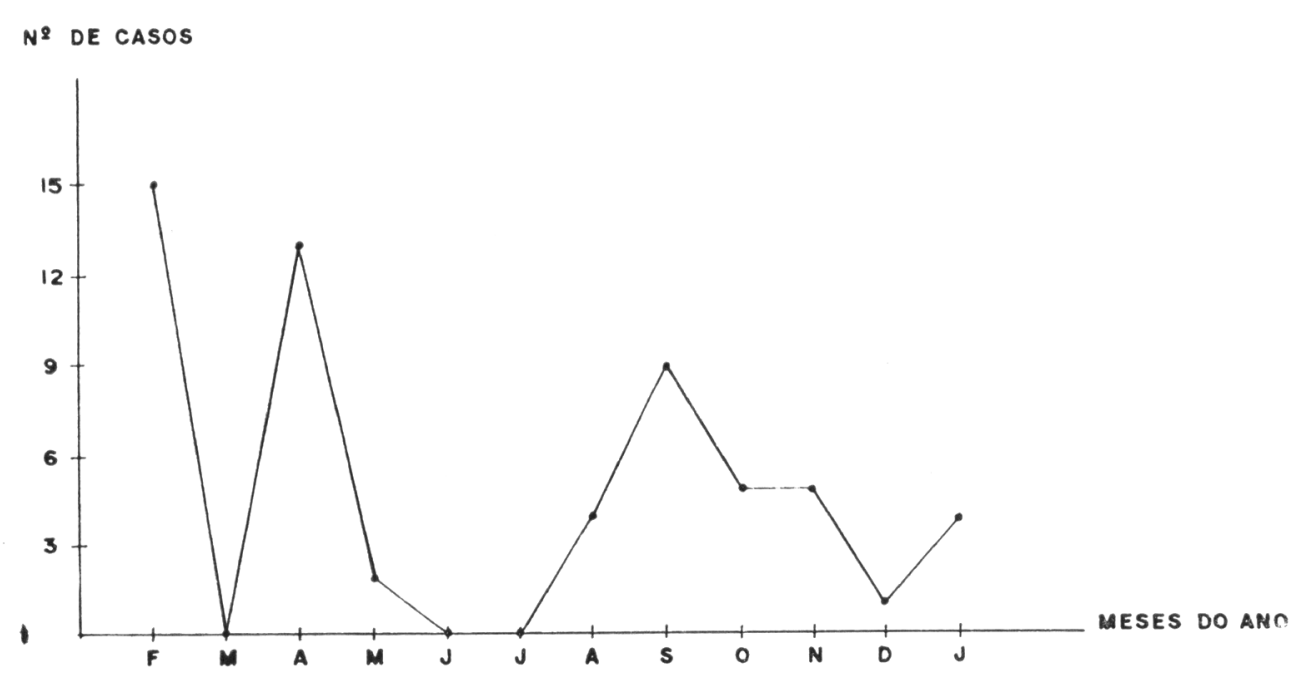

Fig. 2 - Casos, em humanos, com lesões sugestivas de escabiose de origem canina. São Paulo, fevereiro de 1975 a janeiro de 1976 .

A parasitose ocorreu em todas as classes sócio-econômicas, como se visualiza na $\mathrm{Ta}$ bela 5 .

\section{f. Observação do parasita:}

Conseguimos observar a presença do parasita em descamações cutâneas de 3 casos humanos, sendo que realizamos exames de raspado de pele em 12 das 58 pessoas que apresentaram lesões sugestivas de escabiose.

\section{g. Tratamento:}

Dezessete dos 58 indivíduos com lesões sugestivas de escabiose de origem canina 
LARSSON, M. H. M. A. Evidências epidemiológ: as da ocorrência de escabiose, em humanos, causada pelo Sarcoptes scabiei (DeGeer, 1778) vi: canis (Bourguignon, 1853). Rev. Saúde públ., S. Paulo, 12:333-9, 1978.

T A B E L A 5

Casos em humanos com lesões sugestivas de escabiose de origem canina. São Paulo, fevereiro de 1975 a janeiro de 1976.

\begin{tabular}{ccc}
\hline $\begin{array}{c}\text { Classe } \\
\text { sócio-econômica }\end{array}$ & no de casos & $\%$ \\
\hline A & 15 & 25,8 \\
B & 14 & 24,2 \\
C & 10 & 17,3 \\
D & 19 & 32,7 \\
\hline Total & 58 & 100,0 \\
\hline
\end{tabular}

informaram, posteriormente, que após tratamento específico, preconizado por dermatologistas, apresentaram remissão dos sintomas em alguns dias. $\mathrm{Na}$ maioria dos casos, o tratamento foi realizado com especialidades farmacêuticas à base de benzoato de benzila.

\section{DISCUSSÃO}

A sarna sarcóptica em cães ocorreu com frequiência de $51,8 \%$ em fêmeas e $48,2 \%$ em machos, o que nos leva crer que sua incidência independe do sexo (estatisticamente não significante ao nivel de $\alpha=0,05$ ).

Confirmando uma observação anterior (Larsson e col.13, 1974), verificamos que os animais de faixas etárias mais jovens são mais suscetiveis que os adultos, embora tenhamos observado caso de um animal com 12 anos e outro com 19 anos de idade.

Os resultados referentes à variação sazonal da sarna sarcóptica em cães não foram conclusivos, principalmente devido ao número limitado de casos estudados, assim como à pequena flutuação da temperatura ambiental durante 0 ano, em nosso meio.

A incidência de humanos com lesões semelhantes às da escabiose canina foi maior em indivíduos do sexo feminino $(63,8 \%)$ do que nos do sexo masculino
$(36,2 \%)$ (estatisticamente significante ao nivel de $\alpha=0, u 5$ ), observaçao esta condızente com a de rrice ${ }^{18}$ ( 1 yb $\angle$ ). 'I al acnado pode ser explicado, em parte, pelo tato das mulneres serem, mals comumente, responsáveis pelo tratamento dos anımais e, portanto, mass suscetivess a adquirir a parasitose.

Individuos de todas as faixas etárias mostraram-se suscetiveis a apresentar lesões sugestivas de escabiose canina, à semeIhança do que observaram Charlesworth e Johnson: (1974).

A incidência de humanos com lesões dermatológicas semelhantes às da escabiose canina foi bastante variável, ora apresentando frequiências elevadas (fevereiro, abril e setembro), (ra mostrando-se nula (março, junho e jullu': Nossas observaçōes discordaram ias de Mellanby ${ }^{14}$ (1943), Baker e Wharton 1 (1952) e Hornstein citado jur Hermann e Human 12 (1969), que afirmarc'tn ser a escabiose humana mais prevalente no inverno e outono do que no verão. As baixas incidências obtidas por nós, tenham, talvez, sofrido a influência de fatores outros tais como: início das aulas em março; exames e férias escolares, respectivamente em junho e julho; e exames escolares e festas de fim de ano em dezembro, ocasiões em que 0 atendimento ambulatorial também diminui. Além disto, devemos lembrar que em nosso meio a caracterização das estações do ano não é marcante.

Lesões cutâneas sugestivas de escabiose canina foram constatadas em indivíduos de todos os niveis sociais. Em decorrência deste achado cremos que a pobreza e a falta de higiene não são fatores tão importantes na disseminação da doença, como defenderam Epstein 10 (1955); Bopp e Bakos $^{4}$ (1967) e Chen e Dudgale 7 (1972).

A dificuldade de encontrar o agente em exames de raspados de pele humana foi uma constante nos trabalhos de Emde ${ }^{9}$ (1961); Beck $^{3}$ (1965); Tannenbaum 22 
LARSSON, M. H. M. A. Evidências epidemiológicas da ocorrência de escabiose, em humanos, causada pelo Sarcoptes scabiei (DeGeer, 1778) var. canis (Bourguignon, 1853). Rev. Saúde públ., S. Paulo, 12:333-9, 1978.

(1965); Smith e Claypoole 20 (1967) e Charlesworth e Johnson ${ }^{6}$ (1974). Contudo, conseguimos visualizar o parasita em descamações cutâneas de 3 casos humanos, dos 12 nos quais foram feitos raspados de pele. A observação do agente nestes 3 casos foi possivel desde que realizada na fase inicial do processo; tal achado sugere que o $S$. scabiei var. canis é, realmente, parasita capaz de infestar o homem.

Embora Pessoa e Martins ${ }^{17}$ (1974) tenham feito referências à observação de alguns casos humanos refratários ao tratamento especifico das lesões escabiosas com benzoato de benzila, a maioria dos indivíduos do nosso estudo, portadores de lesões cutâneas sugestivas de escabiose canina, apresentaram remissão da sintomatologia alguns dias após o inicio do tratamento com especialidades farmacêuticas à base de benzoato de benzila.

\section{CONCLUSOEES}

1. De um conjunto de 143 pessoas expostas à infestação, $58(40,56 \%)$ apresentaram lesões cutâneas sugestivas de escabiose canina.

2. A incidência de lesões dermatológicas sugestivas de escabiose canina é mais freqüente nos individuos do sexo feminino do que nos do sexo masculino.

3. Lesões cutâneas sugestivas de escabiose canina são passiveis de atingir indivíduos de todas as faixas etárias, indiferentemente.

4. A observação do parasita em lesōes humanas é possivel desde que realizada no inicio do processo.

\section{AGRADECIMENTOS}

Ao Prof. Dr. Ruy Laurenti pela orientação.

LARSSON, M. H. M. A. [Epidemiologic evidence on the ocurrence of scabies in humans, caused by Sarcoptes scabiei (DeGeer, 1778) var. canis (Bourguignon, 1853)] Rev. Saúde públ., S. Paulo, $12: 333-9,1978$.

ABSTRACT: The human infestation with Sarcoptes scabiei var. canis was studied. From 143 humans which were in close contact with 27 infested animals, the author was able to detect 58 infested individuals (40.56\%). The incidence of this zoonosis was higher among women than men and individuals of all ages were indiscriminately affected. The scabies agent was observed in 3 out of 12 pacients submitted to skin scraping.

UnITERMS: Scabies. Dogs. Sarcoptes scabiei.

REFERENCIAS BIBLIOGRAFTCAS

1. BAKER, E. W. \& WHARTON, G. W. An introduction to acarology. New York, Macmillan, 1952.

2. BAKER, E. W. et al, A manual of parasitic mites of medical or economical importance. New York, National Pest Control Association, 1956.
3. BECK, J. A. L. Animal scabies affecting man. Arch. Derm., 91:54-5, 1965.

4. BOPP, C. \& BAKOS, L. Retorno da escabiose no Rio Grande do Sul. An. bras. Derm., 42:1-14, 1967.

5. BUELL, B. G. Difficult to diagnose definitely. Mod, vet. Pract., 54:87, 1973. 
LARSSON, M. H. M. A. Evidências epidemioló gicas ua ocorrêncıa de escabiose, em humanos, causada pelo sarcoptes scabiei (DeGeer, 1778) var. canis (Bourguıgnon, 18๖s). Ktv. saúae publ., S. Paulo, 12:333-9, 1978 .

6. CHARLESWORTH, E. N. \& JOHNSON, J. L. An epidemic of canine scabies in man. Arch. Derm., 110:572-4, 1974.

7. CHEN, S. T. \& DUDGALE, A. E. Morbidity pattern amongst some primary school entrants in Malaysia. Trop. geogr. Med., 24:269-74, 1972.

8. ELGART, M. L. \& HIGDON, R. S. Canine scabies: report of a family outbreak. Sth. med. J., 65:375-6, 1972.

9. EMDE, R. N. Sarcoptic mange in the human. Arch. Derm., 84:633-6, 1961.

10. EPSTEIN, E. Trends in scabies. Aroh. Derm., $71: 192-6, \quad 1955$.

11. FRIEDMAN, $R$. The story of scabies: animal scabies - its relationship to humans. Urol. cutan. Rev., $42: 513-30$, 1936.

12. HERMaNN, W. P. \& HUMAN, H. Seasonal morbidity variation in scabies. Hautarat, 20:467, 1969.

13. LARSSON, M. H. M. A. et al. Ocorrência das sarnas demodécica e sarcóptica em cães do Município de São Paulo. Atual, vet. 3:36-41, 1974.

14. MELLANBY, K. Scabies. London, Oxford University Press, 1943.

15. MULLER, G. H. \& KIRK, R. W. Small animal dermatology. Philadelphia, W. B. Saunders, 1969.
16. NORINS, A. L. Canine scabies in children. Amer, J. Dis. Child., 117:239-42, 1969.

17. PESSOA, S. B. \& MARTINS, A. V. Parasitologia médnca. 9. el. Rio de Janeiro, Guanabara Koogan, 1974.

18. PRICE, I. The scabetic problem. Canad. mea. Ass. J., 66:445-7, 1952 .

19. RICHARDSON, B. D. Sarcoptes scabiei the almost forgotten parasite. Lancet, 1:839, 1972.

20. SMITH, M. E. \& CLAYPOOLE, C. T Canine scabies in dogs and in humen. J. Amer, med. Ass., 199:95-100, 1967.

21. SVARTMAN, M. et al, Epidemic scabies and acute glomerulonefritis in Trinidad Lancet, 1:249-51, 1972.

22. TANNENBAUM, M. H. Canine scabies in man. J. Amer. med. Ass., 193:321-2, 1965.

23. THOMSETT, L. R. Mite infestations of man contracted from dogs and cats. Brit. med. J., 3:93-5, 1968.

24. TOOMEY, N. Scabies of animal origin. Urol. cutan. Rev., 26:473-89, 1922.

Recebido para publicasăo em 28/02/1978 Aprovado para publicasão em 13/04/1978 